\title{
Higgs and SUSY searches at future colliders
}

\author{
ROHINI M GODBOLE \\ Centre for Theoretical Studies, Indian Institute of Science, Bangalore 560012, India
}

\begin{abstract}
In this talk, I discuss some aspects of Higgs searches at future colliders, particularly comparing and contrasting the capabilities of LHC and next linear collider (NLC), including the aspects of Higgs searches in supersymmetric theories. I will also discuss how the search and study of sparticles other than the Higgs can be used to give information about the parameters of the minimal supersymmetric Standard Model (MSSM).
\end{abstract}

Keywords. Higgs; supersymmetry; colliders.

PACS Nos 12.15.-y; 14.80.-j; 14.80.Cp; 14.80.Bn; 14.80.Ly

\section{Introduction}

The SM has been tested to an unprecedented accuracy over the past few decades culminating in the precision measurement at LEP as well as the observation of WW production at LEP-II [1]. The agreement with the SM predictions of the precision measurements at LEP as well as that of the WW cross-section at LEP-II proves that the SM is described by a renormalizable, $\mathrm{SU}(2) \times \mathrm{U}(1)$ gauge field theory. The renormalizability of the theory requires the spontaneous symmetry breaking (SSB) and, in the currently accepted theoretical dogma, Higgs mechanism. However, the direct search for the elusive Higgs has only resulted in lower limits which give $m_{h}>89.7 \mathrm{GeV}[2,3]$.

Thus at present we have no 'direct' proof that Higgs mechanism is 'the' mechanism for the SSB. Further, quantum field theories with fundamental scalars require some mechanism to stabilize the mass of the Higgs $m_{h}$ around the scale of the electroweak (EW) symmetry breaking. Unless the $\mathrm{TeV}$ scale gravity obviates the problem itself [5], supersymmetry is the best available option for the purpose [6]. Since the 'Raison d'etre' for future colliders is to establish the mechanism of the SSB, it is clear that 'Higgs and supersymmetry search' is the most important aspect of physics at the next generation colliders.

In view of the importance of these two searches there exist a large number of discussions of the phenomenological and experimental possibilities in the context of future colliders in literature [7-16]. In this talk, I will focus on some of the recent developments and questions in the Higgs search, viz.

1. In view of the LEP-II results what can Tevatron (and of course LHC) do for Higgs search? 
2. If 'a' scalar is found at LHC how well can one decide that this scalar is 'the' SM Higgs $h$ ?

3. What is the LHC reach for MSSM SUSY Higgs search?

4. If and how does NLC improve the situation?

5. What can $\gamma \gamma$ (and further $\left.\mu^{+} \mu^{-}\right)$colliders do?

As far as supersymmetry (SUSY) is concerned, it is clear that it is broken, albeit the breaking should be at the TeV scale if SUSY has anything to do with particle physics. However, there is no 'real' understanding how SUSY is broken. So, what we really need to do with the SUSY particles (after finding them) is to measure their properties accurately and use them to study how SUSY is broken and learn something about the high scale physics from the way it is broken. It is by now clear that both the LHC and NLC can find TeV scale SUSY, if it exists. The emphasis of all the recent studies has been on how to test different models for high scale physics once SUSY is found.

The future colliders that I would discuss would be mainly Tevatron (Run II, TEV33), LHC and the NLC. The specifications of a future linear collider are not yet completely finalized. The normally considered energies are $\sqrt{s}_{e^{+} e^{-}} \lesssim 500 \mathrm{GeV}$, with luminosities $\sim 20-50 \mathrm{fb}^{-1}$. However, linear colliders with energies extended up to $2 \mathrm{TeV}$ are considered and normally used integrated luminosities for them are usually scaled up to compensate for the $1 / \mathrm{s}$ factor in the cross-section. The technical feasibility of a $500 \mathrm{GeV}$ linear collider is now established [9]. There are also discussions about constructing a $\gamma \gamma$ collider using backscattered laser photons from an $e^{+} e^{-}$machine. Particle production in real scattering of 'real' high energy photons using backscattered laser photons has been observed for the first time [17]. In principle, in the $e^{+} e^{-}$option of the linear colliders (LC's) one has to worry about new phenomena such as Beamstrahlung as well as the backgrounds caused by high energy $\gamma \gamma$ interactions [18]. But they are under control for $e^{+} e^{-}$colliders up to $\sqrt{s}=1 \mathrm{TeV}$ [19]. The high degree of polarization possible at the LC's is particularly useful in precision studies of SUSY.

\section{Higgs search}

\subsection{Theoretical mass limits}

SM Higgs: In the SM only the couplings of Higgs with matter and gauge particles are predicted, but nothing much is known theoretically about its mass, apart from the limits. The upper limit comes from triviality considerations [20,21], i.e., by demanding that the Landau pole in Higgs self coupling $\lambda$ should not occur up to an energy scale $\Lambda$. The lower bound [22] comes from instability of vacuum under fluctuations. The latter is valid only if the Higgs content is minimal viz. a single doublet. Both the bounds depend on $m_{t}$. Given the fairly accurate knowledge on the mass of the top quark, this then gives predictions for both the bounds. The upper bound, in addition, depends on the uncertainties in nonperturbative dynamics which needs to be employed while analysing the large $\lambda$ region. Figure 1 shows the bounds obtained in a recent analysis [23]. This tells us that should we 


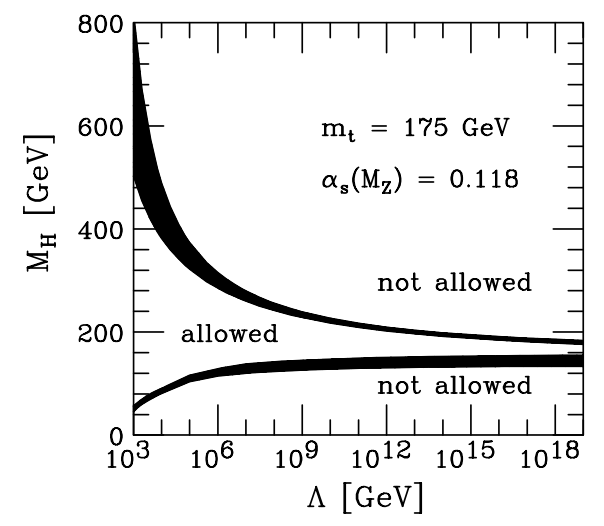

Figure 1. Theoretical bounds on $m_{h}$ in the SM [23].

discover at Tevatron-run II some direct evidence of a Higgs with mass $\sim 115-120 \mathrm{GeV}$, we can take that as an indication that the desert between the weak scale and the Planck scale is sure to be populated and hence also that of possible new physics within the reach of LHC experiments.

SUSY Higgs: Before embarking upon a discussion of the search prospects of the SM Higgs at future colliders, let us also summarize a few things about the predictions of the properties of the various scalars that exist in supersymmetric theories. The scalar sector is much richer in these theories and there are five scalars: three neutrals out of which two are $\mathrm{CP}$ even states: the lighter(heavier) one being denoted by $h_{0}\left(H_{0}\right)$ and one $\mathrm{CP}$ odd state denoted by $A$ and a pair of charged Higgs bosons $H^{ \pm}$. The masses of all the scalars are not independent. They are given in terms of two parameters, which can be chosen either to be $m_{A}, \tan \beta$ or $m_{H^{ \pm}}, \tan \beta$. Here $\tan \beta$ is the ratio of the vacuum expectation values of the neutral members of the two Higgs doublets that exist in the MSSM. As a result of the supersymmetry these masses satisfy certain sum rules and hence inequalities at tree level:

$$
m_{h_{0}} \leq m_{Z}, m_{H_{0}}>m_{Z}, m_{H^{ \pm}}>m_{W}, m_{h_{0}}<m_{H_{0}}, m_{H^{ \pm}} .
$$

In the decoupling limit [24] $\left(m_{A} \rightarrow \infty\right)$ one finds that, independent of $\tan \beta$, all the four heavy scalars become degenerate and infinitely heavy and the mass of the lightest scalar approaches the upper bound. In this limit the couplings of the $h_{0}$ to matter fermions and the gauge bosons approach those of the SM Higgs $h$.

The most interesting, of course, is the upper limit on the mass of the lightest neutral Higgs $h_{0}$ viz. $m_{h_{0}}$. These mass relations receive large radiative corrections, due to the large mass of the top quark $m_{t}$. However, $m_{h_{0}}$ is still bounded. Also note that the corrections will vanish in the limit of exact supersymmetry. The limits on the radiatively corrected scalar masses for the case of maximal mixing in the stop sector are shown in figure 2 [25]. It shows that the mass of the lightest scalar in MSSM is bounded by $\sim 130 \mathrm{GeV}$ even after it is radiatively corrected. This bound does get modified in the NMSSM [26-28]. New results in this context are the two loop calculations [29] of the threshold corrections to the effective quartic couplings of the Higgs potential. These results show that for all 


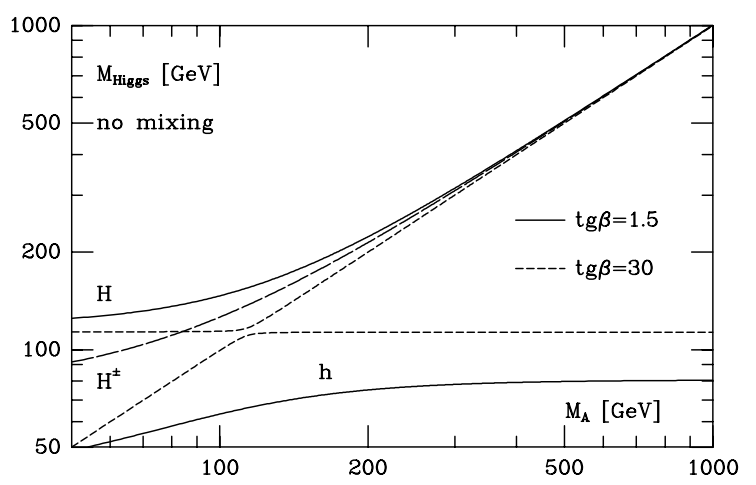

Figure 2. Bounds on the masses of the scalars in the MSSM [25].

reasonable values of the model parameters, $m_{h_{0}}$ is bounded by $\sim 150 \mathrm{GeV}$. The couplings of $h_{0}$ do get modified to some extent by the radiative corrections, but the general features remain the same as the tree level results.

Not only are the scalars much more numerous in the MSSM, their decay patterns are much more involved and depend on the parameters of SUSY model, as both the exact masses and the couplings of the Higgses are dependent on these. Hence the phenomenology of the MSSM scalars is much richer and more complicated than the SM case. Again, calculation of various decay widths including the higher order corrections involving loops of sparticles has been done [30-32].

\subsection{Experimental limits}

The best direct limit on the mass of the Higgs comes from the study $e^{+} e^{-} \rightarrow Z^{*} h$ at LEP-II and will soon reach $108 \mathrm{GeV}[4,33]$. This is already at the limit of the reach of LEP-II. It has been made possible due to the use of $e^{+} e^{-} \rightarrow Z^{*} h \rightarrow \nu \bar{\nu} h$ and the excellent $b$-tagging achieved in the detectors at LEP-II. There exist also, the 'indirect' bounds on $m_{h}$ arrived at from the analysis of precision measurements from LEP [34]. This gives a lower limit on $m_{h}$ of $77 \mathrm{GeV}$ with a $95 \%$ confidence level upper limit of 215 $\mathrm{GeV}$. This then seems to be tantalizingly consistent with the predictions of the bound of $160 \pm 30 \mathrm{GeV}$ from the consistency of the SM. However, it should not be forgotten that new physics within the reach of LHC might change some of the theoretical predictions for the variables used in the precision data. It should be thus borne in mind that with the small expected improvements in the precision, EW data might remain consistent with a somewhat higher upper limit on $m_{h}$. This is to say that it may be possible to relax somewhat the upper bound on $m_{h}$ implied by the analysis [34]. The indirect limits on the mass of the lightest CP even neutral scalar in the MSSM are very similar to that on the SM Higgs, due to decoupling nature of SUSY. The latest (preliminary results on) mass limits for the SUSY Higgses are: $m_{h_{0}}>88.3 \mathrm{GeV} ; m_{A}>88.4 \mathrm{GeV}$ and an absolute limit on $\tan \beta: 0.4<\tan \beta<4.1 \quad(0.7<\tan \beta<1.8)$ for no (large) mixing in the stop sector [4]. 


\subsection{Higgs search at the hadronic colliders}

The mass range for the search of the Higgs divides itself into two regions: i) $102 \leq$ $m_{h} \lesssim 150 \mathrm{GeV}$, ii) $m_{h} \gtrsim 150 \mathrm{GeV}$. The lower limit in (i) is simply a reflection of the current lower limit from 'direct' searches for the Higgs. The upper limit of region (i) is decided by dominant decay modes of the Higgs. A large number of recent discussions [7,8,11,32], both theoretical and experimental, have concentrated on Higgs search strategies in this mass range, for obvious reasons. This is the mass range preferred by the 'indirect' limits; this is also the mass range expected for the lightest supersymmetric Higgs $h_{0}$. It also happens to be the mass range that would be accessible at the Tevatron Run-II/TEV33 as far as the production cross-sections are considered. From the point of view of a clean signal, this happens to be the most challenging range of the Higgs mass as the dominant branching ratio in this case is into the $b \bar{b}$ channel, where the QCD background is about three orders of magnitude higher than the signal.

SM Higgs: Let us first discuss the case of the search for the SM Higgs $h$ at the Tevatron collider. At hadronic colliders, in general, the possible production processes for the Higgs are

$$
\begin{aligned}
g g & \rightarrow h, \\
q \bar{q}^{\prime} & \rightarrow h W, \\
q \bar{q} & \rightarrow h Z, \\
q q & \rightarrow h q q, \\
g g, q \bar{q} & \rightarrow h t \bar{t}, h b \bar{b} .
\end{aligned}
$$

At Tevatron energies, the most efficient processses are the first two of eqs (2). The $W / Z$ produced in association with the $h$, in eqs (2b), (2c), increases the viability of the signal in the $b \bar{b}$ channel. With the use of the high luminosity and a projected improvement in the ' $b$ ' detection, it seems quite likely that the Tevatron might be able to provide a glimpse of the light SM Higgs in the intermediate mass range (IMR). The two new developements here have been the use of $W W^{*}$ channel and/or the use of extra $b^{\prime} s$ in the final state $[35,36]$ and use of neural networks [37] to increase the efficiency of the $b \bar{b}$ channel. Assuming that it will be possible to achieve a $10 \%$ resolution for the $b \bar{b}$ mass reconstruction, an integrated luminosity of $\sim 30 \mathrm{fb}^{-1}$ is required for $3 \sigma-5 \sigma$ signal for $m_{h} \sim 120 \mathrm{GeV}$, when information from all the channels where $h$ is produced in association with a $W / Z$ is combined. For the case of the MSSM Higgs a comprehensive analysis has been done [38].

At the LHC the way out is essentially to use the $h \rightarrow \gamma \gamma$ channel a B.R. which is about 1000 times smaller than that for the $b \bar{b}$ channel but a considerably reduced background. To detect the Higgs in the IMR in this channel the resolution required for $M_{\gamma \gamma}$ measurement is $\lesssim 1 \mathrm{GeV} \simeq 0.1 m_{h}$ [8].

The new developements on the theoretical side have been new calculations of various production cross-sections [32] including the higher order corrections. On the analysis side the new developements have been the detector simulations for the $\gamma \gamma$ mode as well as the $b \bar{b}$ mode. The left panel in figure 3, taken from the CMS/ATLAS techincal proposal [7] shows the expected $S / \sqrt{B}$ for the SM Higgs in the intermediate mass range, using the $\gamma \gamma, b \bar{b}$ modes. The use of $b \bar{b}$ mode for $m_{h}<100 \mathrm{GeV}$ is essentially achieved by using the 

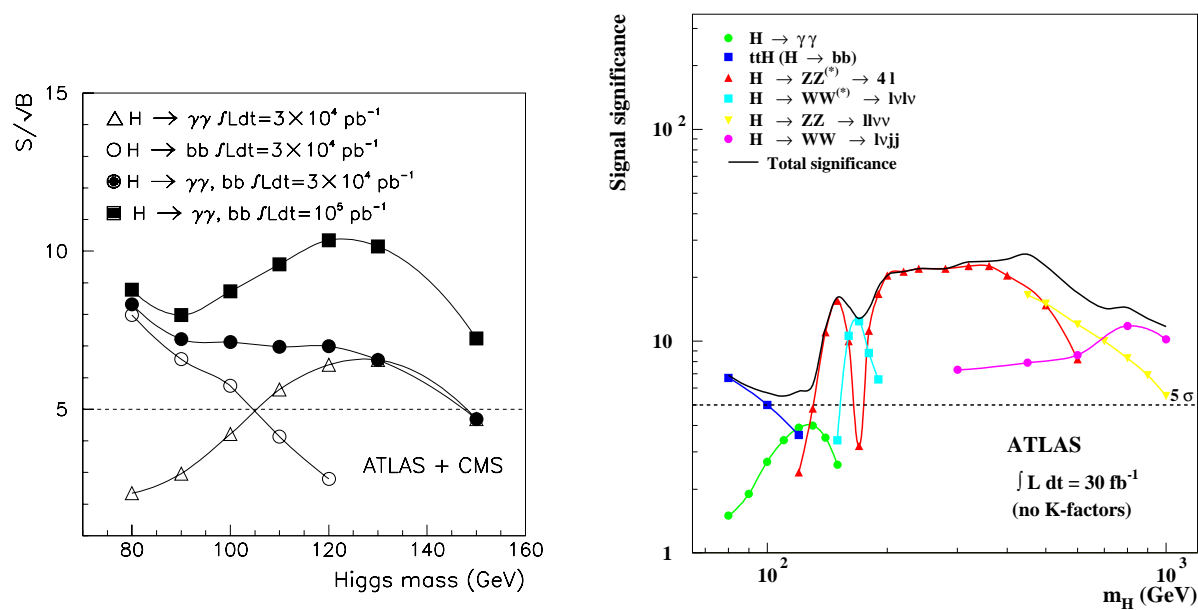

Figure 3. The expected significance level of the SM Higgs signal at LHC. The figure at left is from ref. [7] and the one at right is taken from ref. [8].

associated production of eqs $(2 \mathrm{~b}),(2 \mathrm{c})$. Note that this will require an integrated luminosity of about $30 \mathrm{fb}^{-1}$, which is three years of LHC running at low luminosity. The figure at the right shows the latest analysis of the achievable significance in the ATLAS detector over the entire mass range, taken from ref. [8]. This shows clearly how different mass ranges are covered by different decay modes of the SM Higgs $h$.

A recent development has been a demonstration [39] at the parton level, that the production of the $h$ through the $W W / Z Z$ boson fusion processes of eq. (2d) can be used for Higgs detection using the $\gamma \gamma, \tau^{+} \tau^{-}$and $W W \rightarrow l^{+} \nu l^{-} \nu$ decay modes of the $h$. This has been studied in the context of the ATLAS detector at LHC [40] with a point of view of exploiting this for measuring the relative ratios of the different Higgs couplings at the LHC.

For the mass range (ii) the channels with the higher branching ratio, containing the $V V / V V^{*}(V=W / Z)$ are also the cleanest channels. The figure of merit for a particular channel is clearly the value of the $\sigma \times$ B.R.. Figure 4 taken from ref. [41] shows this for the LHC for both the mass ranges (i) and (ii). The largest contribution to the crosssection here comes from the $g g$ fusion (cf. eq. (2a)). Up to $m_{h}=500 \mathrm{GeV}$ the 'gold plated signal' with four charged leptons arising from the $h \rightarrow Z Z \rightarrow l^{+} l^{-} l^{+} l^{-}$is the cleanest one and has been studied in great detail. In this channel (and of course in the $\gamma \gamma$ channel) the Higgs can be reconstructed as a narrow mass peak. ATLAS collaboration has demonstrated that for $m_{h}=400 \mathrm{GeV}$, with a luminosity of only $10 \mathrm{fb}^{-1}$ (one year of the low luminosity option of LHC), one will see 27 signal events as opposed to a background of $\simeq 10$ events.

The four lepton signature, however, suffers from low branching ratio for $2 m_{W} \lesssim m_{h}<$ $2 m_{Z}$ and requires 30-100 $\mathrm{fb}^{-1}$. In this mass range the channel $h \rightarrow W W\left(W^{*}\right) \rightarrow$ $l^{+} l^{-} \bar{\nu} \nu$ provides a statistically significant signal with a luminosity of $1-2 \mathrm{fb}^{-1}$ [41]. The cross-sections for the associated process of eqs (2b), (2c), are still significant. This can prove useful to see the Higgs in more than one channels (cf. the strategy adopted at the Tevatron).

For $m_{h}>500 \mathrm{GeV}$, detection of the Higgs as a narrow mass peak is no longer feasible 


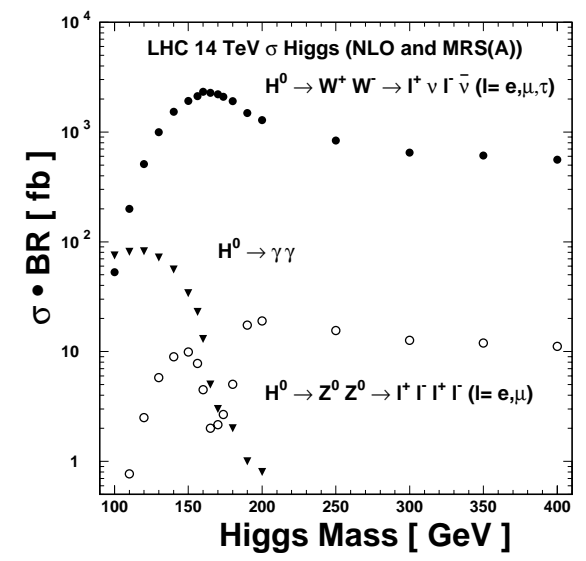

Figure 4. Expected $\sigma \times$ BR for different detectable SM Higgs decay modes [41].

and the size of the four lepton signal is also very small. The best chance for the higher mass Higgs is the detection, by using its production along with forward, high rapidity jets via the process of eq. (2d). For this higher mass range, the detection seems to be a certainty at LHC, up to $m_{h} \sim 700 \mathrm{GeV}$. The processes of eq. (2e) can, in principle, be used to determine the couplings of Higgs to heavier quarks.

MSSM Higgs: The Higgs sector is the one sector of the supersymmetric theories where some discussion can be carried out in a model independent way. For example, upper bound on the mass of $h_{0}$ is quite robust whether we consider MSSM or some extensions of it i.e. the (N)MSSM. The general qualitative observations about the couplings and the mass heirarchy among various scalars in the theory are also model independent. However, the different production cross-sections and the decays do depend crucially on the superparticle spectrum. Hence, while discussing the reach of future colliders, one discusses the SUSY Higgs search in the context of MSSM with certain assumptions about the particle spectrum. For large (small) values of $m_{A}$ the $h_{0}\left(H_{0}\right)$ has mass and couplings similar to the SM Higgs $h$. For MSSM Higgs the discussion of the actual search possibilities is much more involved. For the lightest scalar $h_{0}$ in the MSSM, the general discussions of the intermediate mass SM Higgs apply, with the proviso that the $\gamma \gamma$ branching ratio is smaller for $h_{0}$ and hence the search is much more difficult.

In discussing the search strategies and prospects of the MSSM scalars one has to remember the following important facts:

1. Due to the reduction of the $h_{0} W W$ coupling, the $h_{0} \gamma \gamma$ coupling is suppressed as compared to the corresponding SM case. Of course one also has to include the contribution of the charged sparticles in the loop [31]. The upper limit on $m_{h_{0}}$ implies that the decay mode into $W W(V V)$ pair is not possible for $h_{0}$, due to kinematic reasons. On the other hand, for $H_{0}$ the suppression of the coupling to $V V$ makes the decay less probable as compared to the SM case. As a result, the MSSM scalars are expected to be much narrower resonances as compared to the SM case. For example, the maximum width of $h_{0}$ is less than few $\mathrm{MeV}$, for reasonable values of $\tan \beta$ and even for the heavier scalars $H_{0}$ and $A$, the width is not more than few tens of $\mathrm{GeV}$ even for masses as high as $500 \mathrm{GeV}$. 
2. $h_{0}$ is much narrower than the SM Higgs. However, over a wide range of values of $\tan \beta$ and $m_{A}$, the $h_{0}$ has dominant decay modes into supersymmetric particles. The most interesting ones are those involving the lightest neutralinos, which will essentially give 'invisible' decay modes to the $h_{0}, H_{0}$ and $A$ [30].

3. On the whole for the MSSM scalars the decay modes into fermion-antifermion pair are the dominant ones due to the point (1) above as well as the fact that the CP odd scalar $A$ does not have any tree level couplings to $V V$. Hence, looking for the $\tau^{+} \tau^{-}$ and $\bar{b} b$ final state becomes very important for the search of the MSSM scalars.

As far as the lightest CP even neutral Higgs $h_{0}$ is concerned, the major effects on the search prospects are three :

1. The change in $g g \rightarrow h_{0}$ production cross-section due to the light stop loops. These effects are sensitive to mixing in the stop-sector and so is the mass of $h_{0}$, the latter through radiative corrections.

2. A change in the $h_{0} \rightarrow \gamma \gamma$ width due to light sparticles (specifically stops and charginos) in the loop.

3. Invisible decays of $h_{0} \rightarrow \tilde{\chi}_{i}^{0} \tilde{\chi}_{j}^{0}(i, j=1-4)$.

Many of these have been the subject of detailed investigations of late $[30,31,42,43]$. The sizes of all these effects do depend on the model parameters. All the three effects can conspire together to make the $h_{0}$ 'invisible'. Since both the production and decay are affected by supersymmetric effects, the information is best represented in terms of

$$
R_{g g \gamma \gamma}=\frac{\Gamma^{\mathrm{SUSY}}(h \rightarrow g g) \times B R^{\mathrm{SUSY}}(h \rightarrow \gamma \gamma)}{\Gamma^{\mathrm{SM}}(h \rightarrow g g) \times B R^{\mathrm{SM}}(h \rightarrow \gamma \gamma)},
$$

and

$$
R_{\gamma \gamma}=\frac{B R^{\mathrm{SUSY}}(h \rightarrow \gamma \gamma)}{B R^{\mathrm{SM}}(h \rightarrow \gamma \gamma)}
$$

Figure 5 reproduced from ref. [42] shows the ratio $R_{g g \gamma \gamma}$ as a function of $m_{h}$ and $R_{\gamma \gamma}$ for choices of parameters mentioned in the figure. The depletion in the ratio $R_{g g \gamma \gamma}$ here is mainly due to the small $m_{\tilde{t_{1}}}$. The investigations try to focus on the fact that even though the inclusive $2 \gamma$ signature is substantially reduced the associated production via processes of the eqs (2b), (2c) and (2e) can still provide a viable discovery channel for the light, MSSM Higgs. Also production of Higgs in decays of stops (which are light and hence have large production cross-sections) provides an additional channel.

Luckily the light charginos and neutralinos affect the inclusive $2 \gamma$ channel only in small regions of parameter space [43], once LEP constraints are imposed on the chargino/neutralino sector. The decays of $h$ into invisibles can still contribute to the problem though. Under the assumption of a common gaugino mass at high scale, a dangerous reduction in BR $\left(h_{0} \rightarrow \gamma \gamma\right)$ is possible only in the pathological case of a degenerate sneutrino and chargino. However, for nonuniversal gaugino masses which predict light neutralinos, even after eliminating the region which would give too large a relic cosmological density, there exist regions in parameter space where the usual $\gamma \gamma$ signal for $h_{0}$ drops 

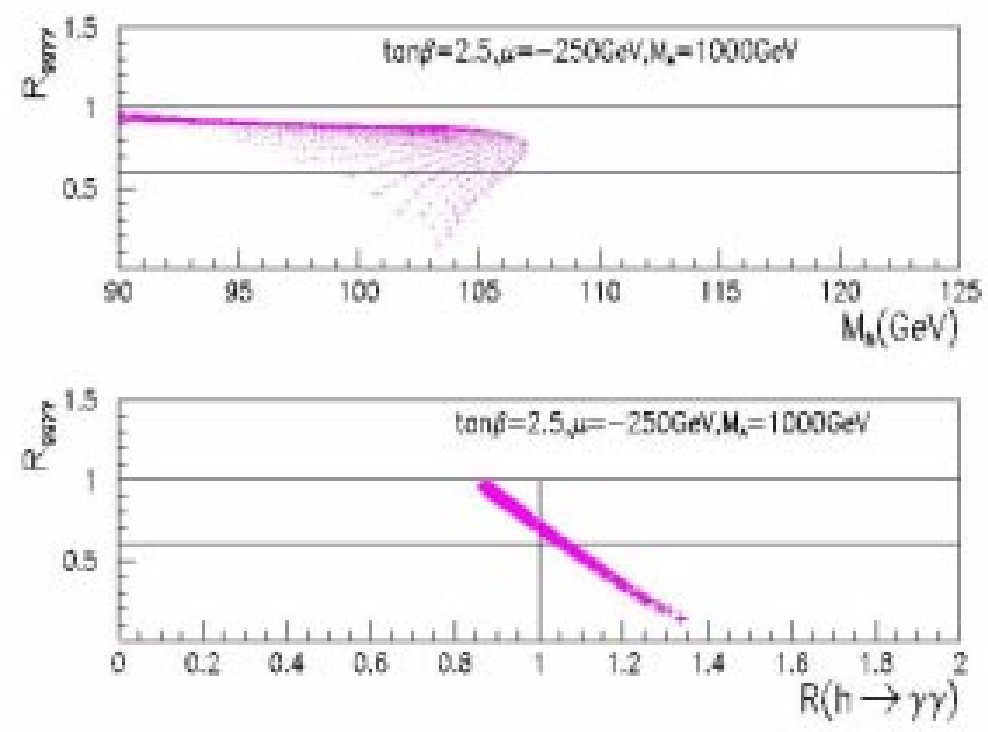

Figure 5. Ratio $R_{g g \gamma \gamma}$ of eq. (3) as a function of $R_{\gamma \gamma}$ of (4) and $m_{h}$ [42]. The values of various parameters are indicated in the figure.

drastically. Figure 6 shows, again for the values of parameters mentioned in the figure caption, $R_{\gamma \gamma}$ as a function of $M_{\chi^{+}}$and $B\left(h \rightarrow \tilde{\chi}^{0} \tilde{\chi}^{0}\right)$. In this case, unlike the case of the light stops, the production of Higgs in decays of charginos/neutralinos does not have very high rates and the search will suffer from the same reduction of the $\gamma \gamma$ channel due to decay into invisibles. Thus in this scenario, for large values of the mass of the CP odd Higgs, a signal for SUSY through the Higgs sector may not be feasible through direct search in the $\gamma \gamma$ channel.

Thus we see that the detection of the lightest SUSY Higgs at Tevatron/LHC will be difficult, but feasible. It will surely require high luminosity run. Recall again here that the low value of $m_{h}$ is the preferred one by the EW measurements [34] and also expected if weak scale SUSY is a reality.

However, since in the MSSM there exist many more scalars in the spectrum one can cover the different regions in the parameter space by looking also for $A, H_{0}$ and $H^{ \pm}$. Figure 7 taken from ref. [8] shows the contours for $5 \sigma$ discovery level for different scalars in the MSSM in the plane of two parameters $\tan \beta$ and $m_{A}$. Thus we see that at low values of $m_{A}$, almost all the scalars of the MSSM are kinematically accessible at LHC. However, at large $m_{A}$, which seem to be preferred by the data on $b \rightarrow s \gamma$ and also by the ever upward creeping lower limit on $m_{h}$ from direct searches, even after combining the information from various colliders (LEP-II, Tevatron (for the charged Higgs search) and of course LHC), a certain region in the $m_{A}-\tan \beta$ plane remains inaccessible. This hole can be filled up only after combining the data from the CMS and ATLAS detector for 3 years of high luminosity run of LHC. Even in this case there exist regions where one will see only the single light scalar. 

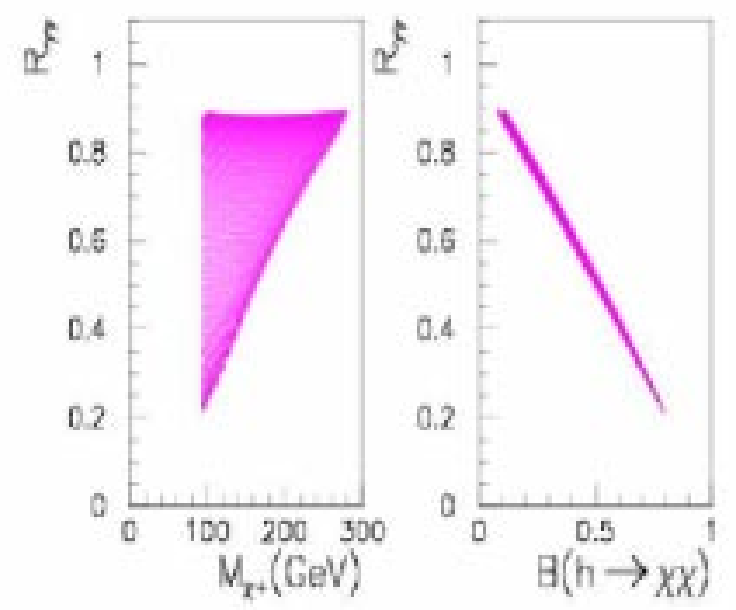

Figure 6. Ratio $R_{\gamma \gamma}$ of eq. (4) as a function of $m_{\chi}^{+}$and the 'invisible' decay width of the $h$ for nonuniversal gaugino masses with $M_{2}=10 M_{1}$, for heavy selectrons [43].

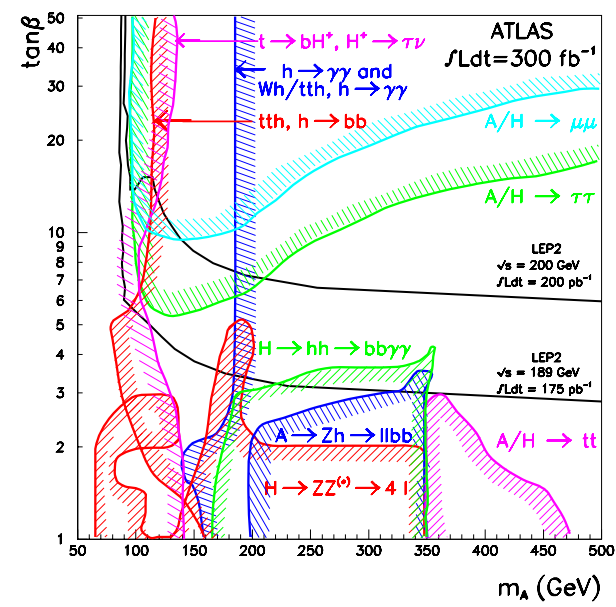

Figure 7. Five $\sigma$ discovery contours for the MSSM Higgs for the ATLAS detector with $300 \mathrm{fb}^{-1}$ luminosities [8].

\subsection{Establishing the quantum numbers of the scalar}

Thus we see that the LHC can see at least one scalar, no matter what its mass. However, to establish such a scalar as the Higgs, one needs to establish two things, viz.

1. The scalar is CP even and has $J^{P}=0^{+}$, 
2. The couplings of the scalar with the fermions and gauge bosons are proportional to their masses.

This is also essential from the point of view of being able to distinguish this scalar from the lightest scalar expected in the MSSM. In general the couplings of the lightest scalar $h_{0}$ are different from the SM Higgs $h$. However, it should be kept in mind that in the large $m_{A}$ region where the mass bound for $h_{0}$ is saturated, these couplings differ very little for the two. As a matter of fact this issue has been a subject of much investigation of late [44,45]. The Snowmass Studies [44] indicate that for a light Higgs $\left(m_{h}=m_{Z}\right)$ such a discrimination is possible only to an accuracy of about $30 \%$. The idea of using the $h / h_{0}$ production via $W W / Z Z$ fusion, to determine the ratios of couplings of the Higgs to different particles, is being studied now [40]. There are also interesting investigations [45] which try to device methods to determine the CP character of the scalar using hadron colliders. It is in these two respects that the planned $e^{+} e^{-}$colliders [9] can be a lot of help.

\subsection{Search of Higgses at $e^{+} e^{-}$colliders}

Even though we are not sure at present whether such colliders will become a reality, the technical feasibility of buliding a $500 \mathrm{GeV} e^{+} e^{-}$(and perhaps an attendant $\gamma \gamma, e^{-} e^{-}$ collider) and doing physics with it is now demonstrated [9]. We will see below that such a collider can play a complementary role and help establish the quantum numbers of the scalar mentioned above. At these colliders, the production processes are $e^{+} e^{-} \rightarrow Z\left(^{*}\right) h$, $e^{+} e^{-} \rightarrow e^{+} e^{-} h, e^{+} e^{-} \rightarrow \nu \bar{\nu} h, e^{+} e^{-} \rightarrow t \bar{t} h$ and similarly the associated production of $h$ with a pair of stops $\tilde{t}_{1} \tilde{t}_{1} h$.

Detection of the Higgs at these machines is very simple if the production is kinematically allowed, as the discovery will be signalled by some very striking features of the kinematic distributions. Determination of the spin of the produced particle in this case will also be simple as the expected angular distributions will be very different for scalars with even and odd parity. For an $e^{+} e^{-}$collider with $\sqrt{s} \leq 500 \mathrm{GeV}$, more than one of the MSSM Higgs scalar will be visible over most of the parameter space [9,32,46]. Even with this machine one will need a total luminosity of $200 \mathrm{fb}^{-1}$, to be able to determine the ratio of $B R(h \rightarrow c \bar{c}) / B R(h \rightarrow b \bar{b})$, to about $7 \%$ accuracy [45]. The simplest way to determine the CP character of the scalar will be to produce $h$ in a $\gamma \gamma$ collider, the ideas for which are under discussion.

At large $m_{A}$ (which seem to be the values preferred by the current data on $b \rightarrow s \gamma$ ), the SM Higgs and $h_{0}$ are indistinguishable as far as their couplings are concerned. A recent study gives the contours of constant values for the ratio

$$
\frac{B R(c \bar{c}) /\left.B R(b \bar{b})\right|_{h_{0}}}{B R(c \bar{c}) /\left.B R(b \bar{b})\right|_{h}}
$$

as well as a similar ratio for the $W W^{*}$ and $b \bar{b}$ widths as a function of $\tan \beta$ and $m_{A}$. As we can see from figure 8 [44], a measurement of this ratio to an accuracy of about $10 \%$ will allow distinction between the SM Higgs $h$ and MSSM Higgs $h_{0}$ up to about $m_{A}=500$ GeV. As stated above, NLC should therefore be able to do such a job. Certainly, the issue of being able to determine the quantum numbers and the various couplings of the scalar including the self couplings forms the subject of a large number of investigations currently $[9,32,47]$. 
NLC Zh Mode: MSSM/SM Ratio Contours

Top Mass = 175 GeV, Higgs Mass = 110 Gev, Max. Mix.

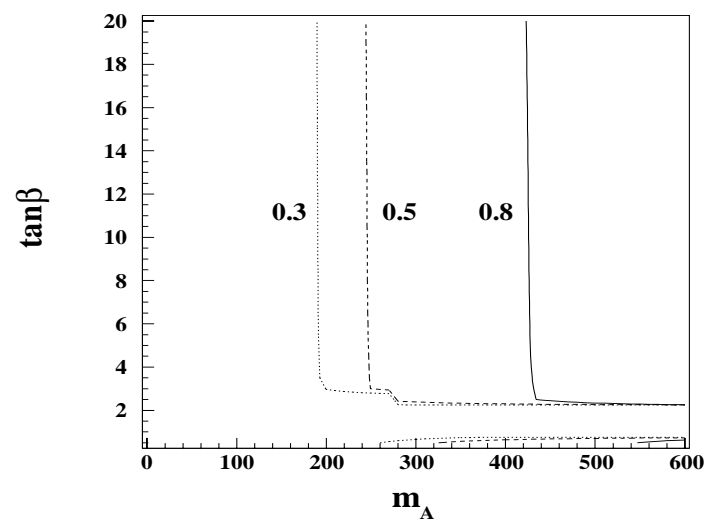

Figure 8. The ratios of relative branching fractions for the MSSM and SM for maximal mixing in squark sector. The specific value of the Higgs mass used is theoretically disallowed at large $m_{A}$ and around $\tan \beta \sim 2$ [44].

\section{Supersymmetric particles other than Higgs}

As explained in the introduction, supersymmetry is the only theoretical framework which can give stabilization of the weak scale against radiative corrections and which has very definite predictions for the presence of additional particles. The search strategies for these sparticles are kind of prototypes for all the searches for physics beyond the SM and are used to define the detector requirements. SUSY has model independent predictions about the spin and electroweak/strong couplings of these sparticles. One has to take recourse to a specific model when we talk about their mass spectrum. They are broadly related to the patterns of SUSY breaking. The ones most often discussed are:

1. Gravity mediated SUSY breaking,

2. Gauge mediated SUSY breaking (GMSB),

3. Anomaly mediated SUSY breaking.

The expected mass patterns and the candidate for the lightest supersymmetric particle (LSP) are different in each case. In the first case the lightest supersymmetric particle is a neutralino $\tilde{\chi}_{1}^{0}$ which is a mixture of Higgsinos and the electroweak gauginos. In the case of GMSB models the LSP is a 'light' gravitino and usually the next lightest supersymmetric particle also behaves like a LSP. In this case, production and decay of the sparticles at colliders produce final states with photons, whereas in the former the final state has $\tilde{\chi}_{1}^{0}$ which may/may not be stable depending on whether the $R$-parity is conserved or violated. In the former case the final state will contain a large amount of missing energy and in the latter case a large number of quarks/leptons. In general the discussion of search for sparticles at the current and future colliders has to cover all these possibilities. In the case of 
gravity mediated SUSY breaking again there are options of considering the constrained, predictive SUGRA framework (where the number of additional parameters of the MSSM goes down from 124 to 5 due to various assumptions) or looking at some model independent aspects. The decay patterns of various sparticles depend crucially on the mass patterns and hence on the assumptions one makes. For example, even in the option of gravity mediated SUSY breaking, there exist virtual LSP's along with $\tilde{\chi}_{1}^{0}$ in some regions of parameter space. Such virtual LSP's can change the phenomenology of the sparticle searches substantially $[48,49]$. A large fraction of the simulation studies done $[14,15]$ so far have been in the context of (M)SUGRA, with a few discussions [50] of the effect of relaxing the assumption of common gaugino mass at the high scale [51] and that of common scalar mass at high scale [52,53] or both and more [54], having started relatively recently.

Before we begin discussions about search strategies for the sparticles, let us note that, the direct searches for sparticles at different $e^{+} e^{-}$and $p \bar{p}$ colliders have so far come up with null results. The only hint of the existence of the sparticles is in the unification of the SU(2), U(1) and SU(3) couplings which happens only in SUSY-GUTS. In this talk I will restrict myself to sparticle searches only in the scenario 1 of SUSY breaking from the above list with and without $R_{p}$ conservation. As already mentioned before, currently the focus of various phenomenological investigations is not so much on the search strategies for sparticles but on the study of how well the soft supersymmetry breaking parameters can be determined once we find the evidence for sparticles. As can be seen from various studies [7-9,14-16] the TeV colliders, viz. Tevatron Run II/ Run III, LHC as well as the NLC should all be able to see the signal for the production and decay of sparticles if the weak scale SUSY is a reality.

\subsection{Discovery potential for SUSY at the different colliders}

At the hadronic colliders the sparticles with largest production cross-sections and hence highest discovery potential, are $\tilde{g}, \tilde{q}$. The $\tilde{g}, \tilde{q}$ are produced via $g g, q \bar{q} \rightarrow \tilde{g} \tilde{g}$ and $g g, q \bar{q} \rightarrow \tilde{q} \overline{\tilde{q}}$. The possible decay modes and various branching ratios clearly depend on the mass spectra. Possible decay modes relevant for the LHC range are $\tilde{q} \rightarrow q \tilde{\chi}_{j}^{0}$ and $\tilde{g} \rightarrow q \bar{q} \tilde{\chi}_{j}^{0}, \tilde{g} \rightarrow q \bar{q}^{\prime} \tilde{\chi}_{i}^{ \pm}$, with $j=1,2,3,4$ and $i=1,2$ depending upon the masses. These will then be followed by further decays of the charginos and heavier neutralinos ending in a $\tilde{\chi}_{1}^{0}$ which is stable for $R$-parity conservation case and will give rise to missing energy in the event. For $R_{p}$ one gets large number of leptons/quarks in unusual combinations due to $\tilde{\chi}_{1}^{0}$ decay, in addition to the other particles in the former case. The cascade decays can give a very characteristic signal with real $Z / W$ 's in the final states if kinematically allowed. Due to the rising importance of cascade decays for the larger masses of $\tilde{g}, \tilde{q}$, a good signal for $\tilde{g}, \tilde{q}$ production and decay is a final state with $m$ jets $(m>0)$, $n$ leptons $(n \geq 0)$ and large missing transeverse energy. The Majorana nature of gluinos can give rise to like-sign-dilepton events. So the expected events are $0 l(1 l)$ : jets, $E_{T}^{\text {miss }}$ and no(1) leptons, SS: same sign dileptons, OSS: opposite side dileptons and $3 l$ : trileptons. For the most commonly expected sparticle mass spectra, the lighter chargions/neutralinos are among the lightest sparticles. These will give rise to the very interesting final state containing only leptons and missing energy via (e.g.) $p \bar{p}(p) \rightarrow \tilde{\chi}_{1}^{ \pm}+\tilde{\chi}_{2}^{0} \rightarrow l^{ \pm} \tilde{\chi}_{1}^{0} Z^{*} \tilde{\chi}_{1}^{0}$. These 'hadronically quiet' trileptons are a very clean channel for SUSY discovery. The associated production of the gauginos is thus signalled by $3 l, 0 j$ : trileptons with a jet 

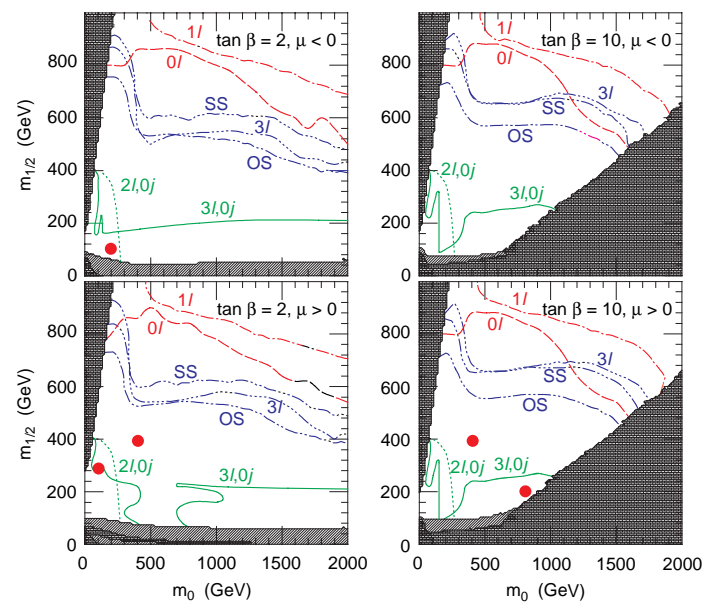

Figure 9. Reach for $S / \sqrt{B}>5$ for various SUSY signatures in SUGRA parameter space. Various symbols are explained in the text. Shaded regions are disallowed either by current searches or theoretical considerations [8].

veto or $2 l, 0 j$ : dileptons with a jet veto. These have been used for SUSY search even at the current Tevatron studies [55]. Higher order corrections to the production cross-sections of the gluinos/squarks and gauginos are now available [56]. The discovery potential of LHC for leptonic channels in the constrained (M)SUGRA scenario is shown in figure 9. The effect of, e.g. nonuniversality in scalar/gaugino masses at high scale, on these analyses can be substantial and is just beginning to be explored. One of the squarks of the third generation viz. $\tilde{t}_{1}$ can be substantially lighter than all the others. As a result, search strategies for the light stop are entirely different $[57,14,15]$. As already mentioned in the discussion of the MSSM Higgs, associated production of $\tilde{t}_{1} \tilde{t}_{1} h_{0}$ can be an interesting discovery channel at the LHC/NLC $[42,58]$.

\subsection{Determination of the soft supersymmetry breaking parameters}

Since production of different sparticles can give rise to the same final states, the real problem at a hadronic collider will be to seperate signals due to different sparticles. The observed signal distributions are sums of products of production cross-sections, branching ratios and acceptances. Hence it seems that a model independent interpretation is impossible. Luckily some kinematical quantities can be extracted in model independent ways using some characteristic decay distributions. Events near the end point of the $m_{l l}$ distribution for three body decay of $\tilde{\chi}_{2}^{0} \rightarrow \tilde{\chi}_{1}^{0} l^{+} l^{-}$(decay caused either by a virtual $Z$ or $\bar{l}$ ) play a very important role in reconstructing the kinematics of $\tilde{g} / \tilde{q}$ cascade decay chain. This can then be used to reconstruct the (M)SUGRA parameters [59]. It is important to investigate model independence of such reconstructions. A study [60-62] shows that the resolution of the $m_{l l}$ distribution end point, depends on the square of the matrix element for the decay and can introduce additional systematic errors in the extraction of SUSY model parameters from kinematics. On the positive side, the decay distributions can give nontrivial information on slepton masses and mixing [62,63]. 

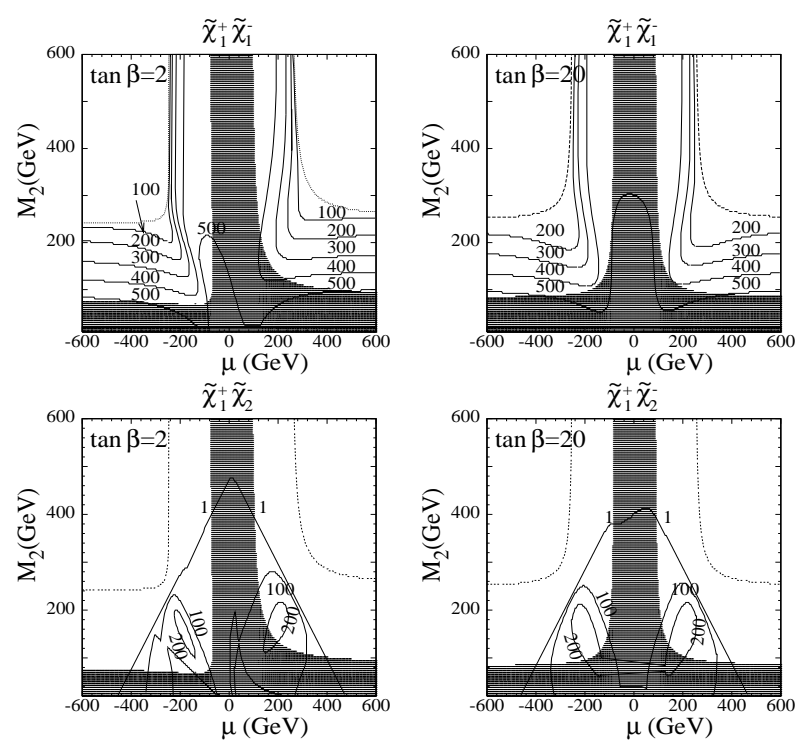

Figure 10. Chargino production cross-sections at an $e^{+} e^{-}$collider with $\sqrt{s}=500$ $\mathrm{GeV}$, with the assumption of a universal gaugino and scalar mass at high scale, with $M_{\tilde{e}_{L}}=M_{\tilde{e}_{R}}=150 \mathrm{GeV}[70]$.

The $\mathrm{TeV}$ energy $e^{+} e^{-}$colliders in planning [9] will play a very useful and complementary role. Of course, only particles with electroweak couplings viz. squarks, sleptons, charginos/neutralinos and Higgses can be produced at these machines. Quite a few detailed studies of the production of the sfermions and chargino/neutralinos exist in literature in the context of $R$-parity conserving SUSY [64-69] as well as in the context of $R_{p}$ SUSY [70]. The cross-sections for the sfermion productions are given completely in terms of their EW quantum numbers and masses, except for those of the third generation where they also depend on the $L-R$ mixing in the sfermion sector, induced by the soft supersymmetry breaking terms. For charginos/neutralinos the cross-sections depend on the SUSY parameters in a nontrivial way. Figure 10 shows contours of constant cross-sections (in fb) for the production of a pair of charginos. The shaded area is ruled out by LEP constraints and the dotted lines show the kinematical limit for chargino production. Thus one sees that the production cross-sections are quite large. In the case with $R_{p}$ conservation, a systematic study of the possible accuracy of the kinematical reconstruction of various sparticle masses and a test of different (M)SUGRA mass relations has been performed. Figure 11 taken from ref. [65] shows the accuracy of the possible reconstruction of the masses of the smuon and the lightest neutralino using kinematic distributions using the slepton production. Using this along with the absolute value of the cross-sections with polarized $e^{+} / e^{-}$beams and angular distributions of the produced sleptons, one can extract the SUSY breaking parameters $M_{2}, \mu$ and $\tan \beta$. It should be then possible to test, 1) the (un)equality of (e.g.) $M_{\tilde{e}_{R}}$ and $M_{\tilde{\mu}_{R}}, 2$ ) as well as the assumption of a common scalar mass at high scale. Figure 12 shows the possible accuracy of reconstruction of $M_{2}^{2}$ and $M_{\tilde{e}_{R}}^{2}-M_{\tilde{e}_{L}}^{2}$, which can thus test the assumption of universal scalar mass. It should be emphasized here that the directions 

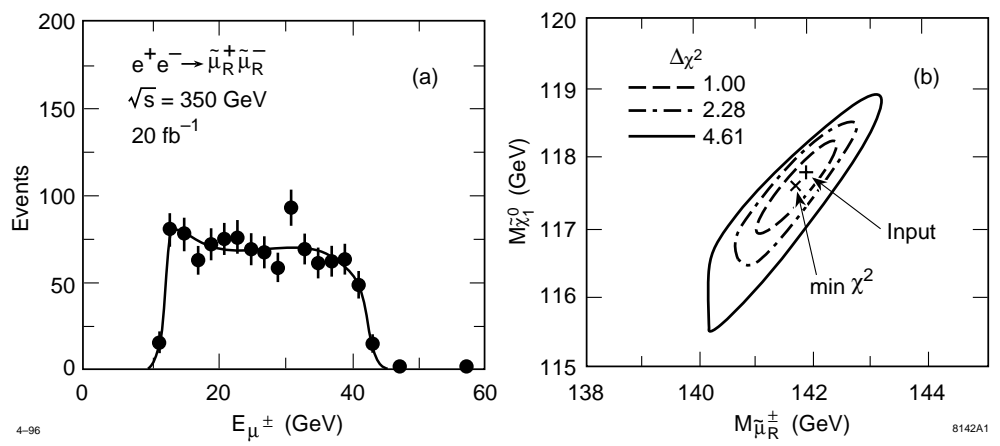

Figure 11. An example of the possible accuracy of the determination of the smuon mass and neutralino mass, for a $e^{+} e^{-}$collider with energy and luminosity as mentioned in the figure [65].

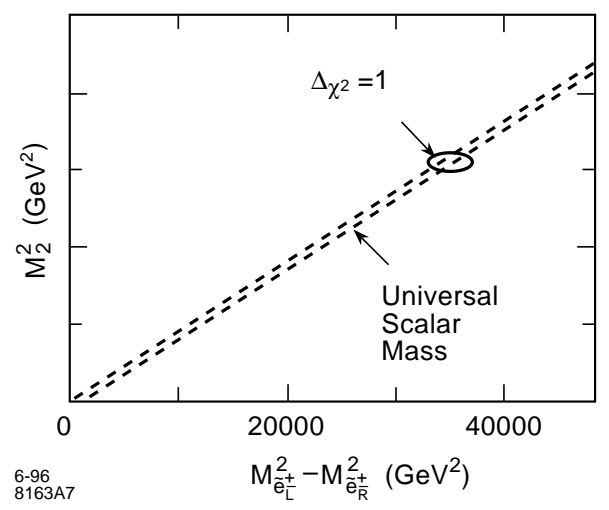

Figure 12. Possible accuracy of a test of universal scalar mass possible at NLC with $\sqrt{s}=350 \mathrm{GeV}$ and luminosity $20 \mathrm{fb}^{-1}$ from the measurements of masses of $\tilde{e}_{R}$ and $\tilde{e}_{L}[67]$

of search for SUSY at the NLC will be largely defined by what we find at the LHC. Relating the measurements of $M_{2}, M_{1}$ etc. at the NLC with the results at the LHC, will then allow us to arrive at an understanding of the soft supersymmetry breaking parameters.

It has been recently demonstrated [71] how using polarized $e^{+} / e^{-}$beams and studying angular distributions in chargino production one can reconstruct the parameters of the chargino sector. Issues under discussion now are also effects of higher order QCD corrections [72-74] on the production and decay of squarks, on the accuracy of kinematic determination of squark mass [75] or possibility of using the highly precise measurements at NLC with polarization to test the equality of fermion-fermion-gauge boson and fermion-sfermion-gaugino couplings [68,76]. 
Thus in summary, we see that LHC will certainly be able to see evidence for sparticles. With clever use of kinematic distributions it seems possible to reconstruct different soft SUSY breaking parameters, first reconstructing various masses kinematically. Along with the NLC one should be able to disentangle different contributions at a hadronic collider from each other and determine the soft SUSY breaking parameters.

\section{Conclusions}

1. The current experimental information from LEP and LEP-II as well as the direct measurement of the top mass indicates that a light Higgs boson is likely. LHC should be able to see a Higgs boson close to $2 M_{W}$ threshold reasonably easily. Heavier ones are also easily detectable up to about $600 \mathrm{GeV}$. In the intermediate mass range between the LEP limit and $2 M_{W}$ one would require the high luminosity, but the Higgs signal would be clear.

2. With the current information and constraints from LEP as well as $b \rightarrow s \gamma$, the decoupling scenario is becoming more and more likely for the SUSY Higgs. At least one Higgs (and in some region of the parameter space two Higgses) can be seen at LHC. However, there still exists a region in the $\tan \beta-\mu$ plane, where the detection of Higgs signal is very difficult if not impossible.

3. Discrimination between a SM and a MSSM Higgs using only LHC seems difficult.

4. LHC can see signals for all non-Higgs sparticles if they exist at mass scales expected in the weak scale SUSY.

5. An $e^{+} e^{-}$collider with $\sqrt{s} \geq 350 \mathrm{GeV}$ can effectively see at least two of the five Higgses, if they are within kinematic reach, independent of any other parameter. The parity of the scalar produced can be trivially determined at an $e^{+} e^{-}$collider. It seems quite difficult to determine the $C P$ character of the scalar produced using only hadronic and $e^{+} e^{-}$colliders. $\gamma \gamma$ colliders might be needed for that. At a $\mu^{+} \mu^{-}$ collider, separation between $h$ and $h_{0}$ based on measurements of relative branching ratios is possible up to $m_{A}=500 \mathrm{GeV}$.

6. Using special kinematical features of the decay distributions, it seems possible (though it needs much more study) to determine some of the soft SUSY breaking parameters even at a hadronic collider. However, a TeV scale $e^{+} e^{-}$collider along with LHC can indeed afford a very clear determination of the soft SUSY breaking parameters if the sparticles are kinematically accessible.

\section{Acknowledgements}

I wish to thank the organisers of the XIII DAE symposium for very efficient organization. I wish to acknowledge the Department of Science and Technology, India and the National Science Foundation's U.S. India Cooperative Exchange Program for partial support under the NSF grant INT-9602567.

Pramana - J. Phys., Vol. 54, No. 4, April 2000 


\section{References}

[1] See for example, talk by A Gurtu in these proceedings

[2] LEP WG for Higgs Boson Searches, CERN-EP/99-060, 1999

[3] Indications from the latest results from the LEP collaborations [4] is that this limit will creep up to $108 \mathrm{GeV}$

[4] Aleph 2000-028, Delphi 2000-050, L3 Note 2525, OPAL Technical Note TN646; Contributions of the LEP collaboration to 'Recontres de Moriond', Les Arcs, France, March 11-25, 2000

[5] See for example, talk by J Maharana, Pramana - J. Phys. 54, 629 (2000)

[6] For one of the earliest discussions see, R Kaul, Phys. Lett. B109, 19 (1982)

[7] CMS Technical proposal, CERN/LHCC/94-38 (1994); ATLAS technical proposal, CERN/LHCC/94-93 (1994)

[8] ATLAS Technical Design Report 15, CERN/LHCC/99-15 (1999)

[9] ECFA/DESY/LC Physics Working Group, A Accomando et al, Phys. Rep. 299, 1 (1998)

[10] Perspectives on Higgs Physics I and II edited by G Kane (World Scientific, Singapore, 1997)

[11] For example see, Run II Higgs Working Group (Run II SUSY/Higgs Workshop) http://fnth37.fnal.gov/higgs.html

[12] The Higgs Working Group Report, Physics at TeV Colliders (Les Houches), A Djouadi et al, hep-ph/0002258

[13] Perspectives on Supersymmetry edited by G Kane (World Scientific, Singapore, 1998)

[14] SUGRA Working Group Report, Run II SUSY Working Group (Run II SUSY/Higgs Workshop) http://fnth37.fnal.gov/susy.html

[15] SUSY Working Group Report, Physics at TeV Colliders (Les Houches) http://lappc-th8.in2p3.fr/Houches99/susygroup.html.

[16] M Carenna, R Culbertson, H Frisch, S Eno and S Mrenna, Rev. Mod. Phys. 71, 937 (1999)

[17] D L Burke et al, Phys. Rev. Lett. 79, 1626 (1997)

[18] R M Godbole, Proceedings of Workshop on Quantum aspects of Beam Physics, Jan. 4-Jan. 9, 1998, Monterey, U.S.A., pp. 404-415, edited by P Chen (World Scientific, 1999) hep-ph 9807379

[19] P Chen, T Barklow and M E Peskin, Phys. Rev. D49, 3209 (1994)

[20] M Sher, Phys. Rep. 179, 273 (1989)

[21] M Lindner, Z. Phys. C31, 295 (1986)

[22] G Altarelli and G Isidori, Phys. Lett. B337, 141 (1994) J A Casas, J R Espinoza and M Quiros, Phys. Lett. B342, 171 (1995)

[23] T Hambye and K Riesselmann, Phys. Rev. D55, 7255 (1997); hep-ph/9708416 in ECFA/DESY study on particles and detectors for the linear colliders edited by R Settlers, DESY 97-123E

[24] H E Haber and Y Nir, Nucl. Phys. B335, 363 (1990)

[25] A Djouadi, J Kalinowski and P M Zerwas, Z. Phys. C57, 569 (1993)

[26] G L Kane, C Kolda and J D Wells, Phys. Rev. Lett. 70, 2680 (1993)

[27] J R Espinoza and M Quiros, Phys. Lett. B302, 51 (1993)

[28] P N Pandita, Pramana - J. Phys. 51, 169 (1998) and references therein, in Proceedings of the Workshop on High Energy Physics Phenomenology, V, Pune, India edited by R V Gavai and R M Godbole (Indian Academy of Sciences, Bangalore, 1998)

[29] S Heinemeyer, W Hollik and G Weiglein, Phys. Lett. B455, 179 (1999); Europhys. J. 9, 343 (1999)

[30] A Djouadi, P Janot, J Kalinowski and P M Zerwas, Phys. Lett. B376, 220 (1996)

[31] For a summary of the effects of sparticles see: A Djouadi, Phys. Lett. B435, 101 (1998), hep-ph 9806315 and references therein

[32] M Spira and P M Zerwas, Lectures at the Internationale Universitätwochen für Kern und Teilchen Physik, hep-ph/9803257 and references therein 
[33] I have used here and later the updated numbers since the talk was presented

[34] LEP Electroweak Working Group, CERN-EP-2000/016, 2000

[35] T Han and R J Zhang, Phys. Rev. Lett. 82, 25 (1999)

T Han, A S Turcot and R J Zhang, Phys. Rev. D59, 093001 (1999)

[36] D Choudhury, A Datta and S Raychaudhury, hep-ph/9809552

[37] P C Bhat, R Gilmartin and H B Prosper, Fermilab-Pub-00/006, hep-ph/0001152 v2

[38] M Carena, S Mrenna and C E M Wagner, Phys. Rev. D60, 075010 (1999) hep-ph 9808312 v2 H Baer, B W Harris and X Tata, Phys. Rev. D59, 015003 (1999)

[39] D Rainwater and D Zeppenfeld, Journal of High Energy Physics 12, 5 (1999)

D Rainwater, D Zeeppenfeld and K Hagiwara, Phys. Rev. D59, 14037 (1999)

T Plehn, D Rainwater and D Zeppenfeld, hep-ph 9911385

D Rainwater and D Zeppenfeld, Phys. Rev. D60, 113004 (1999) erratum to appear (hep-ph 9906218 v3)

[40] D Zeppenfeld, R Kinnunen, A Nikitenko and E Richter-Wa̧s, hep-ph 0002036

[41] M Dittmar, ETHZ-IPP PR-98-10, (hep-ex 9901009)

[42] G Belanger, F Boudjema and K Sridhar, Nucl. Phys. B568, 3 (2000) hep-ph 99004348

[43] G Belanger, F Boudjema, F Donato, R Godbole and S Rosier-Lees, hep-ph/0002039

[44] J F Gunion et al, hep-ph/9703330, in Proccedings of the 1996 DPF/PDB Summer Study of 'New directions in high energy physics' Snowmass (1996), Colarado

[45] J F Gunion, hep-ph/9705282 in ref. [10]

[46] A Djouadi, hep-ph 9910449

[47] See for example, A Djouadi, W Kilian, M Muhlleitner and P M Zerwas, hep-ph/0001169

[48] A Datta, M Guchait and M Drees, Z. Phys. C69, 347 (1996) hep-ph 9503431

[49] For example, see S Ambrosanio, in ref. [15]

H Baer, P G Mercadante, X Tata and Yili Wang, hep-ph 0004001

[50] See for example, A Gouvea, A Friedland and H Murayama, Phys. Rev. D59, 095008 (1999) hep-ph/9803481

[51] S I Bityukov and N V Krasnikov, hep-ph 9907257, hep-ph 9903519

[52] A Datta, A K Datta and M K Parida, Phys. Lett. B431, 347 (1998)

[53] A Datta, A K Datta, M Drees and D P Roy, Phys. Rev. D61, 055003 (2000) hep-ph 9907444

[54] H Baer, M A Diaz, P Quintana and X Tata, hep-ph 0002245

[55] H Baer, M Drees, F Paige, P Quintana and X Tata, hep-ph/9906233 v2

[56] M Spira, in [15]

[57] H Baer, M Drees, R M Godbole, J F Gunion and X Tata, Phys. Rev. D44, 725 (1991)

[58] A Djouadi, J-L Kneur and G Moultaka, hep-ph 9910269

[59] I Hinchliffe, F E Paige, M D Shapiro, J Soderqvist and W Yao, Phys. Rev. D55, 5520 (1997)

[60] M M Nojiri, hep-ph 9907530

[61] M M Nojiri and Y Yamada, Phys. Rev. D60, 015006 (1999)

[62] D Toya, T Kobayashi and M M Nojiri, hep-ph 001267

[63] I Iashvili and A Kharchilava, Nucl. Phys. B526, 153 (1998)

[64] T Tsukamoto, K Fujii, H Murayama, M Yamaguchi and Y Okada, Phys. Rev. D51, 3153 (1995)

[65] J L Feng, M E Peskin, H Murayama and X Tata, Phys. Rev. D52, 1418 (1995)

[66] H Baer, R Munroe and X Tata, Phys. Rev. D54, 6735 (1996)

[67] H Murayama and M E Peskin, Ann. Rev. Nucl. Part. Sci. 46, 533 (1996)

[68] M M Nojiri, K Fujii and T Tsukamoto, Phys. Rev. D54, 6756 (1996)

[69] H-U Martyn and G A Blair, hep-ph/9910416

[70] D K Ghosh, R M Godbole and S Raychaudhury, hep-ph 9904233

[71] S Y Choi, A Djouadi, M Guchait, J Kalinowski, H S Song and P M Zerwas, hep-ph 0002033

[72] M Drees and O J P Eboli, EPJ 10, 337 (1999)

[73] A Bartl, U Eberl, S Kraml, W Majerotto and W Porod, hep-ph 0002115 and references therein 
[74] M Drees, O J P Eboli, R M Godbole and S Kraml, in [15]

[75] J L Feng and D E Finnel, Phys. Rev. D49, 2369 (1994)

[76] H-C Cheng, J L Feng and N Polonsky, Phys. Rev. D56, 6875 (1997); D57, 152 (1998)

E Katz, L Randall and S Su, Nucl. Phys. B536, 3 (1999) 